\title{
On the Effects of Interference between heterogeneous Sensor Network MAC Protocols
}

\author{
Daniel van den Akker*, Bart Braem, Chris Blondia \\ PATS research group, Department of Mathematics and Computer Science \\ University of Antwerp - IBBT \\ Antwerp, Belgium \\ \{daniel.vandenakker,bart.braem,chris.blondia\}@ua.ac.be
}

\begin{abstract}
The growing popularity of sensor networks has resulted in the creation of various MAC protocols, each optimized for a different set of application requirements. However, all of these heterogeneous MAC protocols are designed under the assumption that all nodes in the wireless environment use a common MAC protocol. When multiple sensor networks are deployed in the same wireless environment, this assumption no longer holds since different sensor networks will most likely make use of different MAC protocols. This paper investigates the effect of interference between sensor networks using heterogeneous MAC protocols, based on the network performance of these sensor networks. The network performance of TDMA, CSMA/CA, T-MAC and LPL-MAC, a variant of B-MAC, is examined. Test results show that depending on the interfering MAC protocol, the amount of interference and the load of the network, the average energy consumption can increase by $17 \%$ for contention-based protocols and $62 \%$ for TDMA based protocols. Moreover this interference can cause the endto-end data loss to more than double for contention-based protocols while for TDMA protocols the end-to-end data loss can increase from $0.1 \%$ to more than $40 \%$.
\end{abstract}

Keywords-MAC protocol; sensor network; interference; TDMA; CSMA; B-MAC; T-MAC; Performance evaluation

\section{INTRODUCTION}

As sensor networks continue to gain interest both from the academic community and from the industry, they are being used for an increasingly wide array of applications. The diversity of the requirements imposed by these applications combined with the extremely resource constrained nature of sensor networks has resulted in the development of many different MAC protocols for sensor networks over the last few years. Each of these MAC protocols attempts to optimize the operation of the sensor network for a given set of application requirements. Low Power Listening protocols

* Funded by an Aspirant grant of the Fund for Scientific Research Flanders (FWO).

Part of the research leading to these results has received funding from the Agency for Innovation by Science and Technology (IWT)as part of the Symbionets project and from the Institute for Broadband Technology (IBBT) as part of the NGWinets project. for instance, such as B-MAC [1], provide an energyoptimal method of communication for delay tolerant, low-bandwidth sensor network that do not have a fixed topology. Other MAC protocols, such as the protocol proposed by Hurni et al. [2], start from a predefined set of QoS requirements and attempt to minimize the energy consumption as much as possible. Although such optimized protocols allow for a more efficient operation of the sensor network, they operate under the implicit assumption that all nodes in the wireless environment make use of the same MAC protocol and that the sensor network itself is thus deployed in an isolated environment. As the popularity of sensor networks continues to spread, this is however no longer the case and in the near future it will be the rule rather than the exception to find multiple sensor networks deployed in the same wireless environment. In that case, two different sensor networks operating in the same wireless environment will most likely make use of heterogeneous MAC protocols. Since these MAC protocols have not been designed to take the presence of other MAC protocols into account, they are incompatible and as a consequence they will interfere with each other. Although interference in sensor networks has been well-studied [3] [4], these studies focus on 'external' sources of interference such as interference from WiFi networks. However, to the best of our knowledge, there are currently no studies into the effects of interference that exists between two sensor networks that are based on the same radio technology but that use incompatible MAC protocols.

This paper investigates the effects of interference, caused between sensor networks using heterogeneous MAC protocols, on the network performance of these heterogeneous sensor networks. A comparison is made between the network performance of a sensor network operating without any outside interference and the network performance of the same sensor network operating in a wireless environment in which another sensor network, using a different MAC protocol, is also operating. The focus lies on sensor networks with 
a static deployment operating along the node-to-sink communication paradigm and several popular sensor networking MAC protocols are considered. Network performance is evaluated using Castalia [5], a sensor network simulator. Two different metrics are considered: energy consumption and the reliability of the network. The remainder of this paper is organized as follows. In Section II the examined MAC protocols are presented. Section III proposes the simulation setup and the network performance metrics. Sections IV and $\mathrm{V}$ discuss the effects of interference on each of the considered metrics individually, while in Section VI the overall effects of interference are discussed. A conclusion is provided in Section VII.

\section{Considered MAC PRotocols}

In this work, four sensor network MAC protocols are taken into consideration: CSMA/CA, LPL-MAC, T-MAC and TDMA. These MAC protocols have been selected because they use energy conserving techniques that are often reused or combined in other MAC protocols. LPL-MAC and T-MAC, for instance, are both contention-based MAC protocols but while LPLMAC relies on channel polling to conserve energy, TMAC makes use of a coordinated sleeping schedule to achieve the same goal. A 'generic' TDMA protocol (see section II-C) is used to represent the class of time-division based MAC protocols. Despite its poor energy efficiency, CSMA/CA MAC is also included. In research it is often used as a simple MAC protocol on which new higher-layer protocols can be tested or as a 'reference' case to which other MAC protocols can be compared.

The LPL-MAC T-MAC and TDMA MAC protocols are discussed in more detail in the remainder of this section.

\section{A. LPL-MAC}

The second MAC protocol that is examined is a LowPower-Listening MAC protocol. LPL protocols form an important class of contention-based MAC protocols for sensor networks since they allow energy to be conserved by turning off the radio without the need to create and maintain a synchronized sleeping schedule amongst the nodes of the network. B-MAC, the first LPL-MAC protocol, was introduced by Polastre et al [1]. In B-MAC a node keeps its radio off for most of the time but polls the wireless channel for activity every 'wakeup-interval'. If activity is detected, it will keep its radio enabled in order to receive a packet. If no activity is detected or after a certain time no packet has been received, the node goes back to sleep-mode. When sending a packet, a node will prepend the actual packet with a preamble that is at least as long as the wakeup-interval in order to ensure that the receiving node has enough time to wake up, detect that the channel is active and consequently receive the packet.

For this work we originally wanted to use the B-MAC protocol as it is described in [1]. The original version of B-MAC however, is based on the CC1000 radio chip which allows frames to be sent byte-per-byte and therefore allows preambles of arbitrary length to be used. As with more recent radio chips, the radio simulated by Castalia requires packets to have a limited packet size and a fixed-length preamble. Our implementation therefore differs from the original B-MAC protocol and closely resembles the LPL-MAC protocol that is shipped with TinyOS 2.1.0. As in the original B-MAC protocol, nodes will regularly poll the wireless channel for activity. When a nodes wishes to send a packet it will first attempt to send it using CSMA/CA. If no acknowledgement is received, it assumes that the receiving node is in sleep mode. It will then continue to transmit the same packet over and over in a so called packet-train until it either receives an acknowledgement or a timeout occurs. In order to avoid confusion between our implementation and the original B-MAC protocol, our implementation is referred to as LPL-MAC in the remainder of this paper.

\section{B. T-MAC}

T-MAC [6] and its predecessor S-MAC [7] are contention-based MAC protocols that make use of coordinated sleep schedules between the nodes of the sensor network in order to conserve energy. S-MAC organizes the channel into multiple time frames. Each time frame consists of an active period, during which nodes keep the radio on, and a sleep period, during which the radio is turned off. In order to maintain synchronization between nodes, synchronization messages are broadcasted. The network itself is divided into "virtual clusters' of nodes that adhere to the same schedule. When a node starts up, it listens to the channel for a random time period. If at the end of that period it has not received a schedule from a neighboring node, it chooses its own schedule and starts a new virtual cluster. If, on the other hand, a schedule is received, the node joins the virtual cluster by adopting the received schedule. All nodes are required to periodically broadcast their schedule in order to allow nodes from different virtual clusters to discover each other's schedule. Moreover, each node is also required to adopt to any schedule it receives and to periodically stay awake for the entire time slot in order to discover neighbors using a different schedule. This ensures that neighboring nodes are able to communicate even if they are using different schedules. For regular data communication, S-MAC uses a variant of the the RTS/CTS/DATA/ACK mechanism of 
IEEE 802.11. The main difference is that in S-MAC, the RTS and CTS frames can be used to reserve the channel sending multiple packets whereas in IEEE 802.11 the RTS and CTS frames only reserve the channel for the first data packet. T-MAC improves on the original SMAC protocol mainly by using a variable-length active period. S-MAC uses a fixed-length active period and slot length. As a result it does not cope well with variations in the load of the network. In order to alleviate this problem, T-MAC uses active periods which have a variable duration. Instead of entering sleep-mode after a fixed timeout, a node will only enter sleep-mode if it has not detected an 'activation event' during a certain time period. This timeout period is called the activation timeout period. Activation events include, for example, the reception of a packet, detecting a busy channel or completing the transmission of a data packet. For this work, the T-MAC implementation that is shipped with Castalia is used. This implementation is based on the original T-MAC specification but it addresses a number of practical issues that were not adequately described in the original paper. This T-MAC implementation is described in more detail in [8].

\section{TDMA MAC}

Until now, only contention based MAC protocols have been discussed. Another important class of MAC protocols in sensor networks are the time-division based MAC protocols. Although there are many different Time Division Multiple Access (TDMA) MAC protocols currently available for sensor networks, all of these protocols are based on the same principle where the wireless channel is divided into multiple time slots. The main advantage of using TDMA in sensor networks is that nodes only need to turn their radio on during those time slots that have been assigned to them. Since the radio can be disabled during all other time slots, the energy consumption of the sensor network is reduced significantly. Moreover nodes do not need to contend for channel access, which allows the wireless channel to be used more efficiently. Using TDMA in sensor networks presents a number of challenges. As in traditional TDMA-setups, the TDMA protocol must be able to keep the clocks of the nodes in the network synchronized. Moreover mechanisms are required to allocate the available time slots to the nodes in the network in an intelligent manner. In traditional setups such as cellular networks, TDMA is only used to control channel access over a single hop. In sensor networks however, TDMA is used for multi-hop communication. Moreover the resource-constrained nature of sensor networks needs to be taken into account and TDMA protocols need to be scalable to accommodate sensor networks consisting of hundreds or even thousands of nodes. Although currently numerous mechanisms exist for time synchronization and slot allocation in sensor networks [9] [10], this work does not focus on any specific mechanism. Instead the effects of interference on time-division based MAC protocols in general are investigated. A TDMA MAC protocol that is 'semistatically' configured is therefore considered. In the beginning of each simulation run, the nodes in the network are assigned a configuration that contains the slot assignments of the network. These slot assignments do not change during the simulation and are based on the topology of the network and the routing paths from the nodes to the sink. In order to allow nodes to synchronize their clocks with the sink node of the network, the available time slots are organized into superframes. At the beginning of each superframe, a number of slots are reserved for broadcasting so-called SYNC-packets. Based on these packets, receiving nodes are able to synchronize to the clock of the sending node. In the beginning of each simulation run, each node is assigned a synchronization-parent to which it will synchronize its clock. The synchronization-parents of the nodes in the network are calculated based on the topology of the network. As with the slot assignments, the synchronization-parent of a node does not change during the course of the simulation. Each synchronization-parent is also assigned a time slot at the beginning of each superframe during which it is required to broadcast a SYNC-packet. If a node loses its synchronization with its synchronization parent, it will stop sending data packets and listen to the wireless channel until synchronization is restored.

\section{Methodology}

In this section, the simulations that were used to study the effect of interference between heterogeneous MAC protocols are further discussed. All simulations are performed using Castalia 3.0 [5], a sensor network simulator that is specifically designed to simulate wireless sensor networks and body area networks. It uses an advanced channel model that is able to model interference between sensor nodes accurately. During each simulation run, a wireless environment is simulated in which either one or two sensor networks are deployed. Simulations where only one network is present are used to establish the baseline network performance. When two sensor networks are present, both networks are equipped with different MAC protocols to measure the impact of interference. Each network consists of 100 nodes with the sink node placed at the edge of the network. Nodes are organized along a randomized grid deployment within an area measuring 100 by 100 meters. In order to create interference between the two networks, the geographical areas in which these 
networks are deployed overlap. Moreover, the amount of overlap is varied in order to create several levels of interference. When there is $0 \%$ overlap, both networks are deployed adjacent to each other. In this case interference only take place between the nodes near the shared edge of the networks. When there is $100 \%$ overlap, the deployment areas of the networks coincide and interference occurs between all nodes of both networks. Each simulation run starts with a startup phase. This phase lasts 200 (simulated) seconds and is used to build the connection map for each network. During this phase, nodes broadcast a number of 'hello'-messages that are consequently received by their neighbors. These messages are sent independently from the used MAC protocol. Moreover, in order to avoid interference, the transmission of these messages is timed such that only one message is sent at a time. At the end of this phase, the routes from the nodes to their sink are calculated based on the position of the nodes in the network and the link quality to their neighboring nodes. The reason for using static routes is that the focus of this work is to investigate interference between MAC protocols. By using static routes, the influence from the routing protocol is minimized. If one of the networks is using the TDMA MAC protocol, the synchronizationparents and slot assignments are also calculated at this stage. During the remainder of the simulation, each node regularly generates a data packet and forwards it to the sink of the network. This is done during one hour of simulated time. Data packets are between 123 and 125 bytes large, depending on the size of the MAC header and are forwarded along the static paths calculated at the end of the startup phase. To investigate the influence of the network load, the data generation interval varies between 10 and 60 seconds. At the end of each simulation run, the metrics used to measure networking performance are recorded. Two different metrics are considered: Energy consumption and endto-end data loss. The energy consumption is obtained directly from Castalia and data loss is measured by comparing the number of data packets received by the sink of the network and the number of generated data packets. If a data packet is retransmitted by the MAC layer, this packet is only counted once. In the following sections, the effect of interference on each of these metrics is discussed. To investigate the effects of interference between two heterogeneous time divisionbased MAC protocols, the case where both networks are using the TDMA MAC protocol is also considered. To emulate the heterogeneous nature of these MAC protocols, the calculation of the slot assignments and synchronization parents will not take the presence of the other network into account.

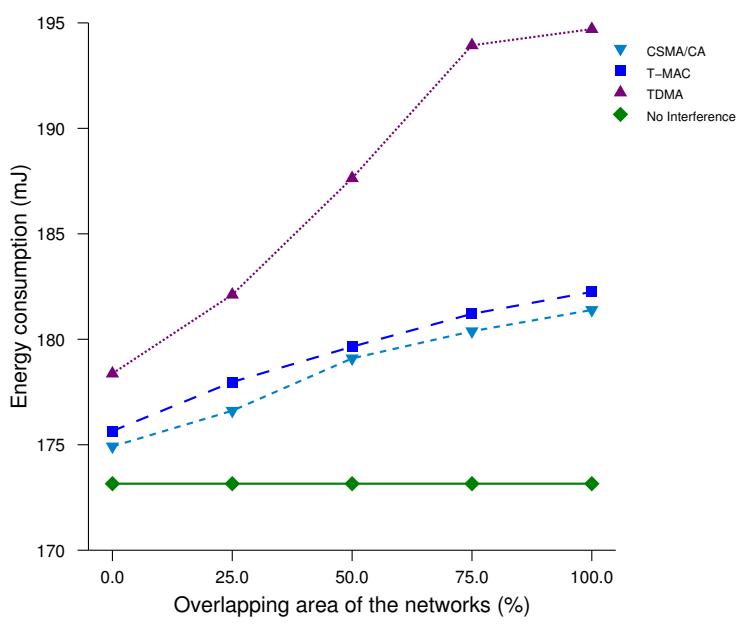

Figure 1. LPL-MAC: Average energy consumption. (Interval=30s)

\section{ENERGY CONSUMPTION}

In this section, the impact of interference between heterogeneous MAC protocols on the average energy consumption in the network is discussed. We only consider the energy consumption of the radio itself and do not take the energy used for packet processing into account. The energy consumption of CSMA/CA is not discussed. This protocol namely requires nodes to keep their radio enabled at all times. Since an enabled radio consumes the same amount of energy regardless of whether a frame is being received or not, the impact of interference on the energy consumption of this MAC protocol is negligible.

\section{A. LPL-MAC}

Figures 1 and 2 display the average energy consumption per node for a sensor network using LPL-MAC. In Figure 1 the energy consumption for a fixed data generation interval and a varying amount of overlap between the networks is displayed while in Figure 2 the data generation interval (and thus the network load) is varied. As with the remainder of the figures in this paper, each data point represents the average value measured over 25 simulation runs. The standard deviation is fairly small but is not shown in order to keep the figures clear. As shown in Figure 1, the energy consumption of LPL-MAC is higher when there is another sensor network also operating in the wireless environment than in the baseline scenario where the LPL-MAC network receives no external interference. Moreover, the impact on the energy consumption increases with the amount of overlap between the networks. This increase is caused by the fact that LPL-MAC nodes are woken up by channel activity generated by the interfering MAC protocol. The impact of the interference received from a contention-based MAC protocol on the energy 


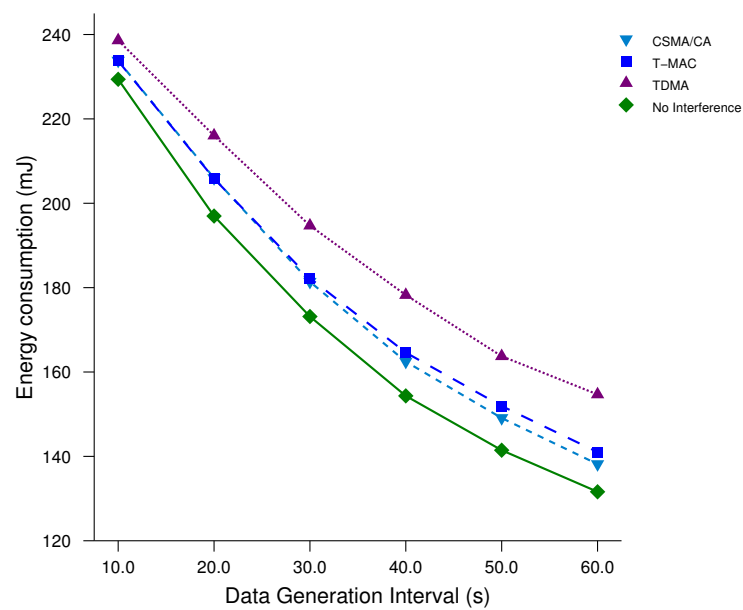

Figure 2. LPL-MAC: Average energy consumption. (Overlap=100\%)

consumption is relatively limited. Even when there is $100 \%$ overlap between the networks, the energy usage of the LPL-MAC network is only increased by $5.5 \%$. TDMA has a larger impact on the energy efficiency of LPL-MAC, since the energy consumption can increase by as much as $12.5 \%$. This is caused by the fact that the considered contention-based protocols will only send packets on the channel when they have actual data to send while nodes in the TDMA network will also send out SYNC-packets on a regular basis to keep the clocks of the nodes in the network synchronized. T-MAC will also occasionally send out synchronization messages, but these messages are sent at a much lower rate than the SYNC-packets of the TDMA protocol. When the impact of interference is considered for different data generation intervals (Figure 2), it becomes clear that while the absolute energy consumption decreases for larger data generation intervals, the relative impact of the interference from other MAC protocols actually increases. In the case where data packets are being generated every 10 seconds, energy consumption is increased by at most $4 \%$ by the presence of another MAC protocol. When a data generation interval of 60 seconds is used, the energy consumption can increase by as much as $17 \%$. This is a result of the fact that an interfering MAC protocol can only wake up an LPL-MAC node if it is in sleep mode while the interfering MAC protocol is active. For higher network loads, the LPL-MAC nodes operate at an increased duty cycle and consequently spends less time in sleep-mode. As a result the effect of interference on energy usage is smaller.

\section{B. T-MAC}

As shown in Figure 3, T-MAC responds in a similar way to varying levels of interference as LPL-MAC. Energy consumption increases with the amount of over-

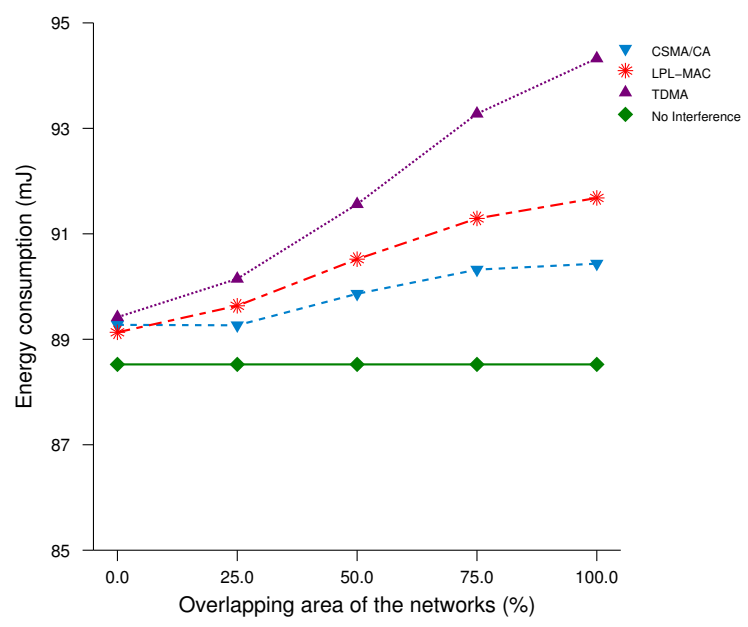

Figure 3. T-MAC: Average energy consumption per node. (Inter$\mathrm{val}=30 \mathrm{~s}$ )

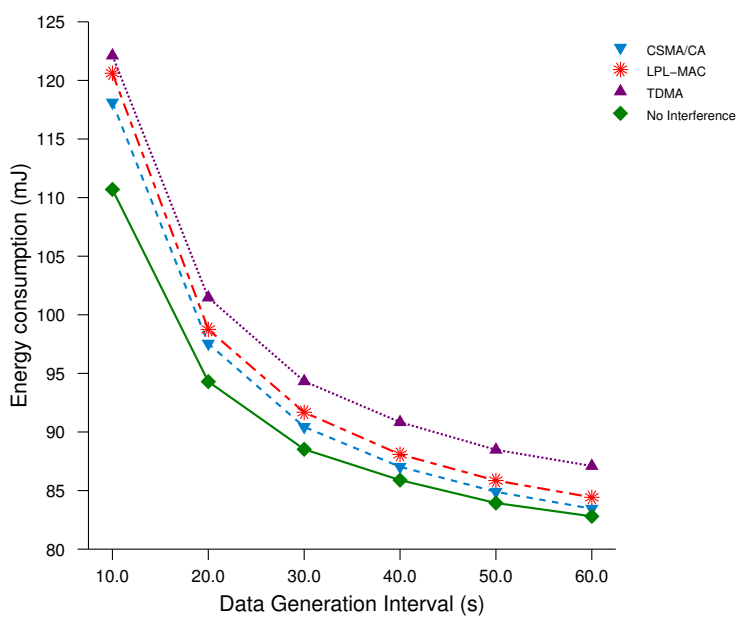

Figure 4. T-MAC: Average energy consumption. (Overlap=100\%)

lap between the networks, and the TDMA MAC has a higher impact than the contention-based protocols. When the interfering network uses a contention-based MAC protocol, energy consumption increases by at most 3.5\%. When a TDMA MAC protocol is used, energy consumption increases by at most $6.5 \%$. As with the LPL-MAC protocol, the increase in energy consumption is caused by idle listening. Although nodes using T-MAC will wake up based on a common sleeping schedule rather than activity on the channel, the amount of time these nodes keep their radios on after waking up is dependent on the activity on the channel. As a result of the increased idle listening time, the energy consumption is also increased. Figure 4 displays the average energy consumption for a sensor network using T-MAC for varying data generation intervals. When a data generation interval of 60 seconds is used, the energy consumption is increased by at most $5 \%$. In the case that data packets are generated every 10 


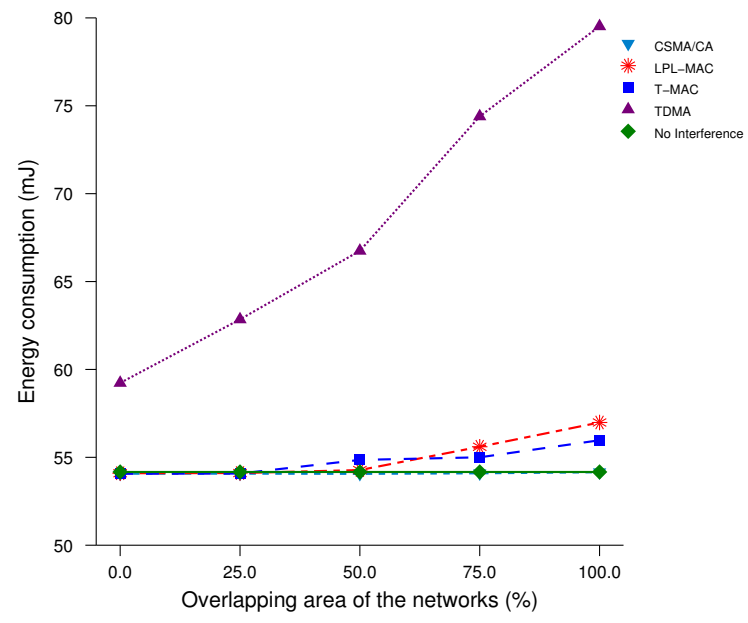

Figure 5. TDMA: Average energy consumption. (Interval=20s)

seconds, energy consumption is increased by more than $10 \%$. This is behavior is explained by the fact that T-MAC will by default use a 'conservative activation timeout'. As discussed in [8], this means that T-MAC will keep the radio enabled for another full activation timeout period every time that the radio perceives that the channel is busy. Since channel activity increases not only with the load of the T-MAC network but also with the load of the interfering network, the impact of interference is, in contrast to LPL-MAC, larger for smaller data generation intervals than for larger intervals.

\section{TDMA}

In Figure 5, the impact of interference on the energy consumption of TDMA is shown for varying levels of overlap between the interfering sensor networks. As this figure clearly shows the presence of a sensor network using one of the contention-based MAC protocols has a negligible effect on the average energy consumption of the TDMA network when there is little or no overlap between these networks. When the amount of overlap between the networks increases, the energy consumption increases with at most 5\%. Since nodes in the TDMA network only take the pre-calculated slot assignments into account when deciding when to turn their radio on or off, this increase in energy consumption is a direct result of TDMA nodes losing synchronization with the rest of the network. Loss of synchronization occurs when a node fails to receive a SYNC-frame four times in a row. The probability that a TDMA node loses synchronization, however, is limited due to the fact that all considered contention-based protocols defer transmission of a packet when they detect that the channel is busy. As the overlap between the networks increases, the number of TDMA nodes experiencing interference from the other sensor network

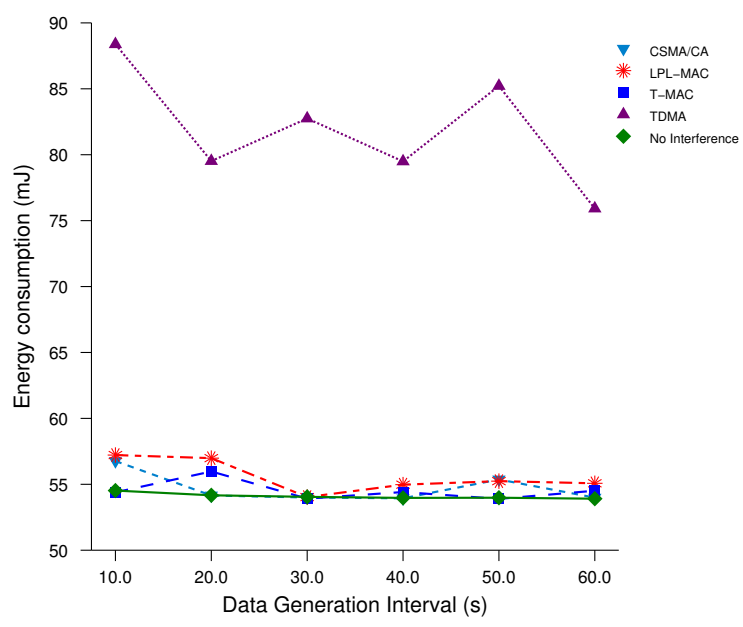

Figure 6. TDMA: Average energy consumption. (Overlap=100\%)

also increases. This in turn increases the probability that a node on the TDMA network loses synchronization which causes the energy consumption to be increased as well. In the case where two TDMA networks interfere with each other, collisions with SYNC-messages occur much more frequently due to the fact that TDMA nodes do not perform carrier sensing before transmitting a packet. Moreover, as discussed in Section III, there is no synchronization between nodes from different networks and the used slot assignments do not take into account the presence of the other TDMA network. This results in a severe increase in energy usage. Moreover the energy usage increases with the overlap between the networks. When there is no overlap, energy consumption is increased by $9 \%$. When both networks fully overlap, energy usage increases with more than $45 \%$. Figure 6 displays the energy consumption of TDMA for different data generation intervals. This figure shows that there is a limited fluctuation of the influence from the contention-based MAC protocols on the energy consumption. They only result in an increase in energy consumption of $1 \%$ to $5 \%$. The largest increases can be observed for the smallest data generation interval. When two TDMA sensor networks interfere with each other, there is a much larger fluctuation in the influence these networks have on each others energy consumption. The increase in energy consumption varies between $41 \%$ and $62 \%$.

\section{ReliabiLity}

This section discusses the influence of interference between heterogeneous sensor networks on the reliability of these networks. Reliability is measured by considering the end-to-end data loss in the network. It should be noted that all contention-based MAC protocols make use of acknowledgements and retransmissions to ensure that packets are delivered. In order to 


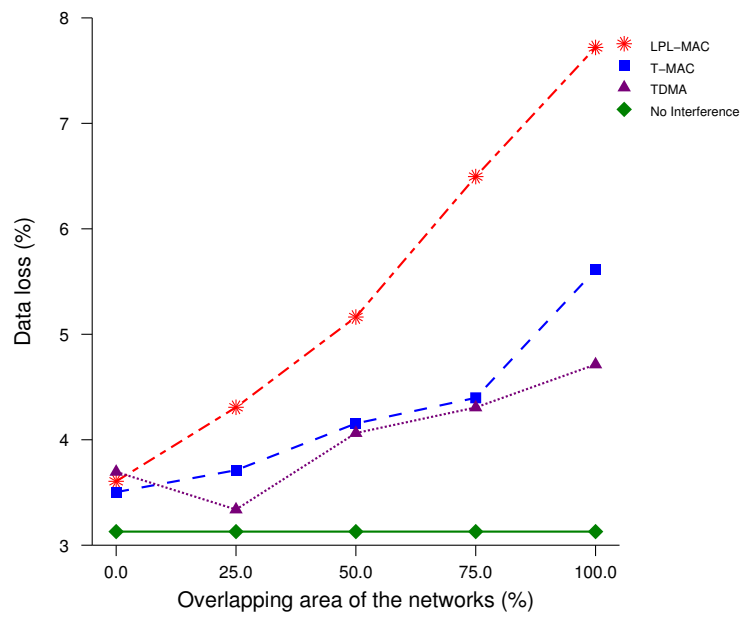

Figure 7. CSMA/CA: Average end-to-end data loss. (Interval=30s)

be able to make a fair comparison between these MAC protocols, the number of retransmissions has been set to two for all contention-based MAC protocols. This is the default value for T-MAC in Castalia. The TDMA MAC protocol does not use acknowledgements and will only transmit each data packet at most one time.

\section{A. $C S M A / C A$}

Figure 7 displays the influence of interference between heterogeneous MAC protocols on the end-toend data loss of a sensor network using CSMA/CA for different amounts of overlap between the networks. As this graph clearly shows, the amount of data loss severely increases with the amount of overlap between the networks. When the two networks are deployed adjacent to each other, the end-to-end data loss only increases from 3\% to at most $4 \%$ as a result of interference. In the case where the two sensor networks fully overlap, the interference from TDMA causes the data loss to jump from 3\% to 5\% while LPL-MAC causes the end-to-end data loss to more than double. The impact of interference on the end-to-end data loss for a varying data generation interval is shown in Figure 8. This graph shows that while the absolute amount of data loss under interference is higher for low values of the data generation interval, the relative increase of data loss, compared to the baseline scenario, is actually lower. When a data generation interval of 10 seconds is used, the relative end-to end data loss is 0.6 to 1.2 times higher than in the baseline scenario, depending on the interfering MAC protocol. For a data generation interval of 60 seconds, the end-to-end data loss is between 0.8 and two times higher. When the effects of interference are compared between the interfering MAC protocols it becomes clear that overall TDMA has a smaller impact on the reliability of CSMA than the contention based MAC protocols. This is most likely

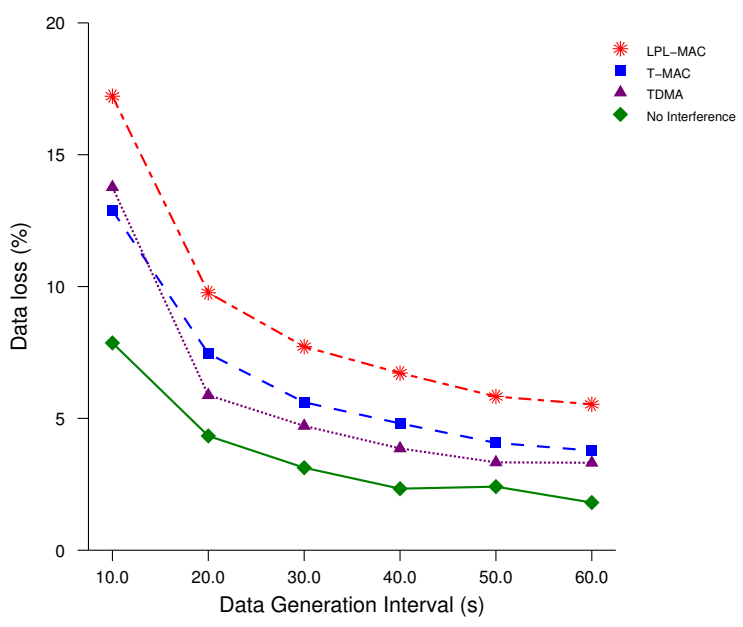

Figure 8. CSMA/CA: Average end-to-end data loss. (Overlap=100\%)

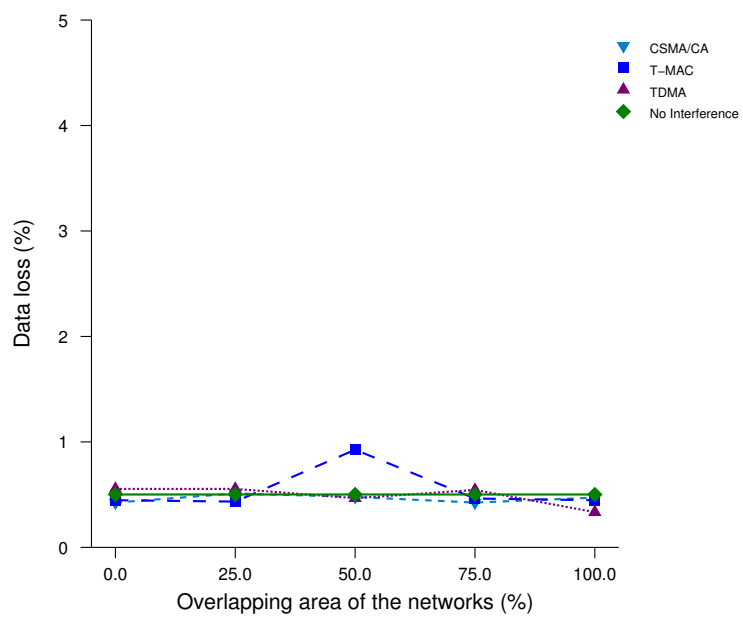

Figure 9. LPL-MAC: Average end-to-end data loss. (Interval=30s)

due to the fact that TDMA will only transmit a data packet once, regardless of its successful reception while the other MAC protocols will, just like CSMA/CA, try to retransmit the packet. Moreover it should also be noted that the impact of LPL-MAC on the reliability is noticeably higher than the impact of the other protocols. This is because in TDMA each data packet is sent at most once whereas in T-MAC each data packet is sent, including retransmissions, at most three times. If a node using LPL-MAC fails to receive an ACK for a packet, however, a packet train is sent in the assumption that the receiving node is still asleep. This means that LPL-MAC performs, in comparison to other MAC protocols, a huge amount of retransmissions before giving up.

\section{B. $L P L-M A C$}

The end-to-end data loss of a sensor network using LPL-MAC is shown for a varying amount of overlap with the interfering network in Figure 9. As this figure 


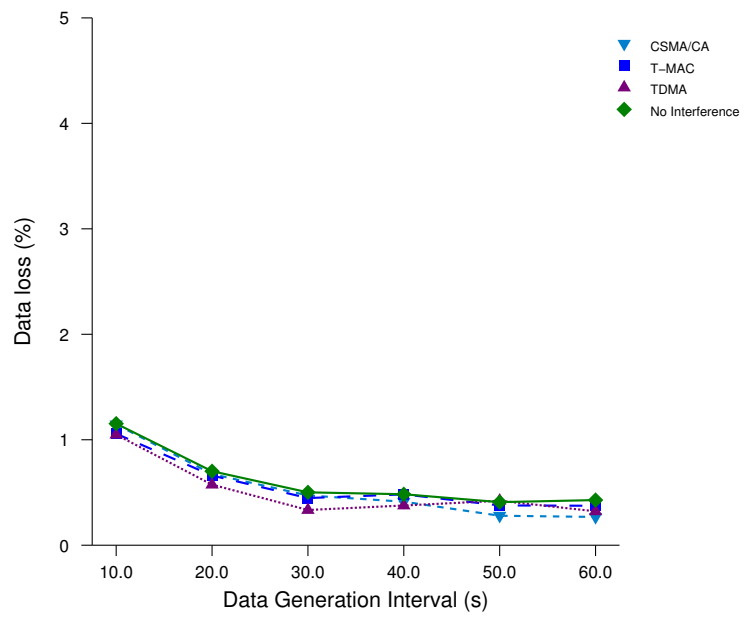

Figure 10. LPL-MAC: Average end-to-end data loss. (Overlap $=100 \%$ )

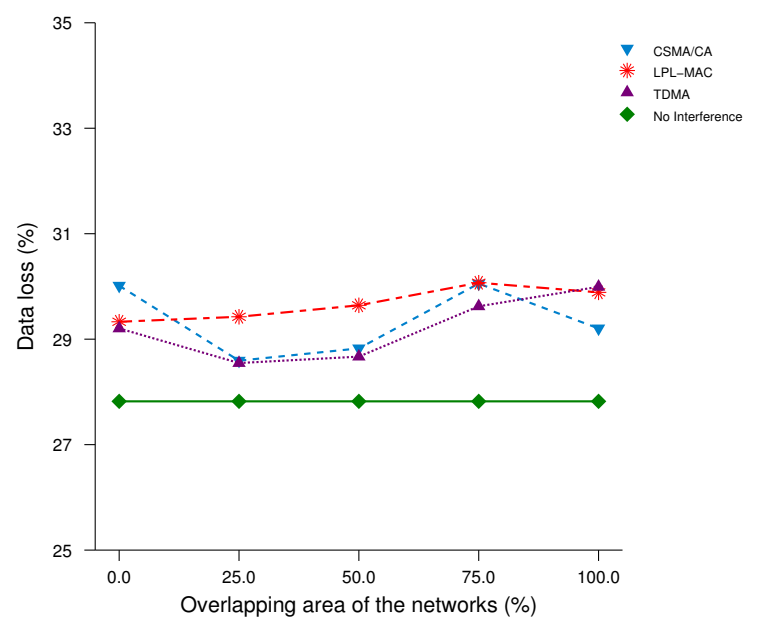

Figure 11. CSMA/CA: Average end-to-end data loss. (Interval=30s)

shows, the reliability of LPL-MAC is largely unaffected by interference from a heterogeneous MAC protocol. Figure 10 shows that this is also the case when the data generation interval is varied. This behavior can be attributed to the low power listening mechanism of LPL-MAC. As explained in Section V-A, the packets in the packet-train used by LPL-MAC can also be regarded as retransmissions of the original packet. This means that, as long as there is sufficient bandwidth available to transmit these packet trains, LPL-MAC is extremely resilient to external interference.

\section{T-MAC}

The end-to-end data loss of the T-MAC protocol, for varying overlap between the two sensor networks is shown in Figure 11. When the end-to-end data loss of T-MAC is considered for the baseline scenario, these measurements show that T-MAC has an end-to-end data loss which is much larger than that of the other

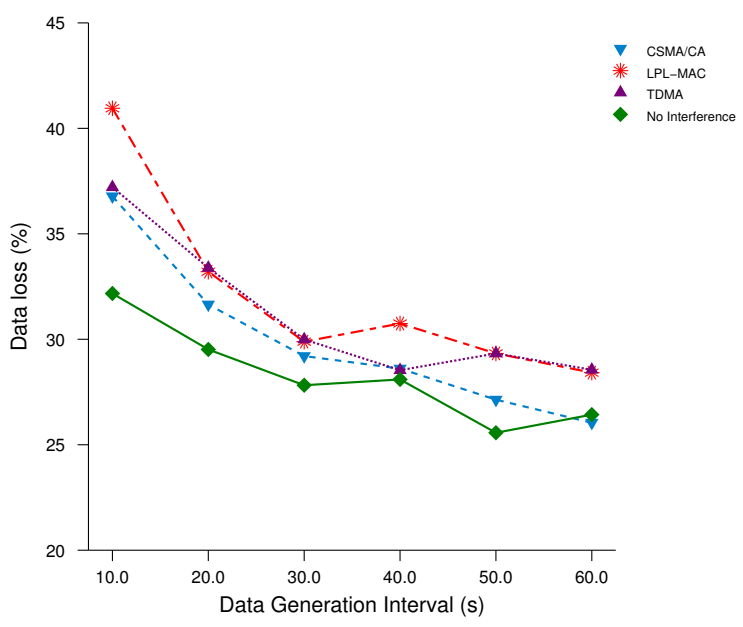

Figure 12. T-MAC: Average end-to-end data loss. (Overlap=100\%)

considered MAC protocols. In the baseline scenario T-MAC displays a data loss of almost $28 \%$, while CSMA/CA and LPL-MAC only suffer $3 \%$ and $0.5 \%$ data loss respectively. This difference may be the result of the 'early sleeping' problem of T-MAC as discussed in [6]. The early sleeping problem arises when most traffic in the network is unidirectional, such as in a node-to-sink scenario. Due to the interaction between the RTS/CTS and activation-timeout mechanism, nodes may go to sleep early while other nodes still have data to send them. Normally this problem should only affect the maximum throughput and not the reliability of the T-MAC protocol. The T-MAC implementation provided by Castalia however, discards a packet after two retransmissions regardless of whether the RTS-CTS handshake failed or no acknowledgement was received for the packet. Moreover, Castalia's T-MAC implementation does not implement the Future-Request-to-Send mechanism discussed in [6], which is able to overcome the early sleeping problem. Despite the fact that the baseline end-to-end data loss is very high, T-MAC seems to be able to cope reasonably well with external interference. Even for the case where there is $100 \%$ overlap between the sensor networks, the end-to-end data loss only increases from $28 \%$ to $30 \%$. When the end-to-end data loss is considered for a varying data generation interval, as shown in Figure 12, it can be observed that although the impact of interference increases for smaller data generation intervals, this impact is still fairly small. Even for a data generation interval of 10 seconds, the end-to-end data loss only increases from $32 \%$ to $41 \%$ and the impact is much smaller for larger data generation intervals. T-MAC is thus resilient towards interference for both high and low network loads. This resilience may be due to the RTS-CTS handshake used by T-MAC. Although this mechanism 


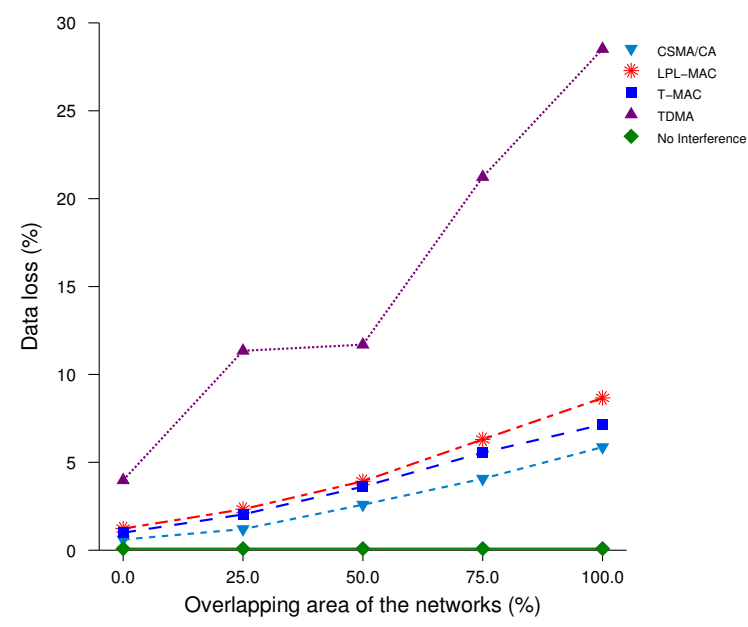

Figure 13. TDMA: Average end-to-end data loss. (Interval=30s)

does not prevent heterogeneous MAC protocols from transmitting during the time that the channel is reserved, this mechanism at least ensures that the channel is clear both at the sender and at the receiver when a packet is transmitted.

\section{TDMA}

Figure 13 displays the influence of the overlap between the interfering networks on the end-to-end data loss of a sensor network using TDMA. The baseline end-to-end data loss is very low and hovers around $0.1 \%$. Since the slot assignments have been calculated to prevent two TDMA nodes of the same network from interfering with each other, the measured data loss is the result of packets-loss on the wireless medium. When the TDMA network is receiving interference from a contention-based MAC protocol, the amount of overlap between these networks has a clear effect on the end-to-end data loss. When the networks are deployed adjacent to each other, there is at most $1.5 \%$ data loss. When the overlap between these networks increases, the effects of interference become larger. When the networks fully overlap, the data loss increases from $0.1 \%$ to between $6 \%$ and $9 \%$, depending on the interfering MAC protocol. This increase in loss is caused by the fact that, despite the collision-avoidance techniques used, collisions with TDMA packets will still occur if the node using a contention-based MAC protocol starts its transmission before TDMA does. When two TDMA MAC protocols interfere with each other the incurred data loss ranges between 5\% and 25\% depending on the overlap between these two networks. Considering the fact that there is practically no data loss in the baseline scenario, in this case the reliability of TDMA is thus heavily impacted. When the reliability is examined for different data generation intervals, as shown in Figure 14, it becomes clear that the impact of

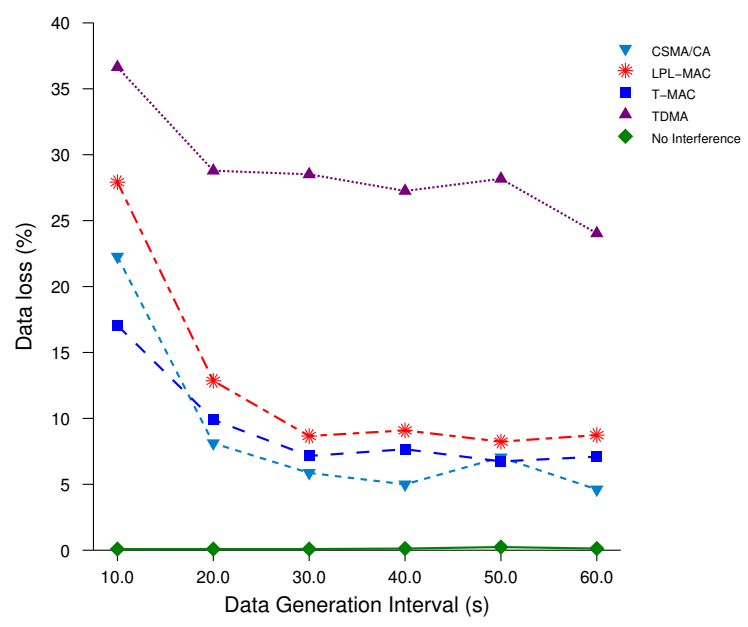

Figure 14. TDMA: Average end-to-end data loss. (Overlap=100\%)

interference is largely the same for all data generation intervals. When the network load is very high, however, the impact of interference becomes more severe.

\section{IMPACT OF INTERFERENCE}

From the figures shown in the Section IV and V it can be concluded that while the interference between two heterogeneous MAC protocols has, in general, a limited effect on the energy usage of these networks, the effect on the end-to-end reliability is much more severe. When the energy consumption is considered, the only test case where the increase in energy consumption is likely to cause any serious issues is the case where two TDMA MAC protocols interfere with each other. As discussed in Section IV-C, the energy consumption can increase with $62 \%$ in this case. Considering the fact that TDMA MAC protocols are often used in statically deployed networks where energy consumption is extremely important, this increase in energy consumption can have a dramatic effect on the network lifetime. In all other test cases, the impact is less severe. Over all considered test scenarios, the energy consumption is increased by at most $10 \%$ under the influence from a heterogeneous contention-based MAC protocol. Moreover these contention-based MAC protocols are also able to cope better with the interference from TDMA than the TDMA MAC protocol itself. Although the impact of interference between heterogeneous MAC protocols on the energy consumption cannot be neglected, in most cases this impact remains relatively small. Unfortunately the effect of interference between heterogeneous MAC protocols on the reliability of these networks is much more severe. If the general effect of this interference is considered, it becomes clear that for most MAC protocols there is a noticeable impact even when there is little or no overlap between the networks. When the networks fully overlap, however, 
the end-to-end data loss is multiple times higher than in the baseline scenario. Of all test cases, the most dramatic impact on reliability is measured in the case where two TDMA MAC protocols interfere with each other. As discussed in Section V-D, the end-to-end data loss can increase to more than $40 \%$ in this scenario. Considering that in the baseline scenario only $0.1 \%$ of all data is lost, such an impact is more than enough to cripple the operation of the sensor network. Even when TDMA is interfered by a contention-based MAC protocol, the end-to-end data loss can still be an order of magnitude higher than in the baseline scenario. Interestingly enough, TDMA does not have the highest impact on the reliability of the contention-based MAC protocols. CSMA/CA for instance experiences on average around two times more data loss under the influence of LPL-MAC than under the influence of TDMA. Moreover LPL-MAC has in most cases the largest impact on the reliability of other MAC protocols. This is because the wakeup mechanism of LPL-MAC, which causes LPL-MAC to remain largely unaffected by interference, causes a lot of additional traffic which negatively impacts the reliability of other MAC protocols. From the results discussed in Section V-C it would seem that the reliability of the T-MAC protocol is also little impacted by interference. However, in the performed experiments T-MAC did have a high end-toend data loss percentage in the baseline scenario due to the early sleeping problem. It is therefore not clear how T-MAC would perform under circumstances where the baseline end-to-end data loss is lower. Although interference between heterogeneous MAC protocols clearly has a profound impact on the end-to-end reliability, the gravity of this reduced reliability depends on the actual application of the sensor network itself. Most sensor network applications that operate along the node-tosink communication paradigm do not require explicit reliability guarantees and allow for a certain amount of data to be lost. However, even the operation of faulttolerant networks will be noticeably impacted when the end-to-end data loss is several times to a magnitude larger than expected.

\section{CONClusion \& Future Work}

In this paper we have provided a performance evaluation investigating the effects of interference between heterogeneous MAC protocols on the network performance. In most cases, the impact of interference on the energy consumption is relatively small. This interference does have a profound impact on the reliability of the involved sensor networks. This means that, as sensor networks become more popular and more sensor networks are being deployed, developers will have to consider the effects of interference between heteroge- neous MAC protocols and take adequate measures to counter these effects. Future work will therefore focus on applying the 'Symbiotic Networking paradigm' to this scenario. The core principle of 'Symbiotic Networking' [11] is that all networks operating in the same wireless environment will cooperate to optimize the network operation globally across these networks. This cooperation is achieved by negotiating on network parameters over the entire network stack and across different networks. By applying this principle here, a TDMA and a T-MAC sensor network could for instance arrange their communication in such a way that the active TDMA slots coincide with the sleeping period of T-MAC. Although applying the concept of symbiotic networks presents a number of challenges, such as how exactly these networks would negotiate, it offers a number of interesting perspectives with regard to future research.

\section{REFERENCES}

[1] J. Polastre, J. Hill, and D. Culler, "Versatile low power media access for wireless sensor networks," in Proceedings of the 2nd international conference on Embedded networked sensor systems. ACM New York, NY, USA, 2004, pp. 95-107.

[2] P. Hurni and T. Braun, "Maxmac: A maximally traffic-adaptive mac protocol for wireless sensor networks," in Wireless Sensor Networks, 7th European Conference, EWSN 2010. Proceedings. Springer, 2010, pp. 289-305.

[3] A. Sikora and V. Groza, "Coexistence of IEEE 802.15.4 with other Systems in the $2.4 \mathrm{GHz}-\mathrm{ISM}-\mathrm{Band}$," in Proceedings of the IEEE Instrumentation and Measurement Technology Conference, 2005. IMTC 2005, May 2005, pp. 1786-1791.

[4] W. Yuan, X. Wang, and J.-P. M. G. Linnartz, "A Coexistence Model of IEEE 802.15.4 and IEEE 802.11b/g," 2007 14th IEEE Symposium on Communications and Vehicular Technology in the Benelux, pp. 1-5, Nov. 2007.

[5] H. N. Pham, D. Pediaditakis, and A. Boulis, "From simulation to real deployments in wsn and back," 2007 IEEE International Symposium on a World of Wireless, Mobile and Multimedia Networks, pp. 1-6, Jun. 2007.

[6] T. van Dam and K. Langendoen, "An adaptive energy-efficient MAC protocol for wireless sensor networks," Proceedings of the first international conference on Embedded networked sensor systems - SenSys '03, pp. 171-180, 2003.

[7] W. Ye, J. Heidemann, and D. Estrin, "Medium Access Control With Coordinated Adaptive Sleeping for Wireless Sensor Networks," IEEE/ACM Transactions on Networking, vol. 12, no. 3, pp. 493-506, Jun. 2004.

[8] Y. Tselishchev, A. Boulis, and L. Libman, "Experiences and lessons from implementing a wireless sensor network mac protocol in the castalia simulator," in $W C N C, 2010$, pp. 1-6.

[9] T. Brooks, H. Bakker, and K. Page, "A Review of Synchronization Methods in Wireless Sensor Networks," FM 2009: Formal Methods, pp. 516-531, 2008.

[10] J. Mao, Z. Wu, and X. Wu, "A TDMA scheduling scheme for many-to-one communications in wireless sensor networks," Computer Communications, vol. 30, no. 4, pp. 863-872.

[11] E. Poorter, B. Latré, I. Moerman, and P. Demeester, "Symbiotic Networks: Towards a New Level of Cooperation Between Wireless Networks," Wireless Personal Communications, vol. 45, no. 4, pp. 479-495, Mar. 2008. 